\title{
La producción académica de los egresados del posgrado de instituciones de educación superior en México
}

\author{
Teresita de Jesús Méndez Rebolledo \\ Universitat de Barcelona. España. \\ tmendere7@alumnes.ub.edu
}

Recibido: 29/3/2016

Aceptado: 24/5/2016

Publicado: 26/10/2017

\section{Resumen}

La sociedad del conocimiento es una transición de las economías industriales a las economías fundadas en el saber, que están basadas en inversiones en educación, formación, investigación y desarrollo. Por ese motivo, interesa analizar la trayectoria de egreso y los perfiles profesionales de los egresados con base en la producción académica, la satisfacción con las competencias adquiridas, el programa educativo y la institución, así como, por medio del análisis estadístico de regresión lineal, dar respuesta a la pregunta siguiente: ¿en qué medida la trayectoria de egreso se distingue por su producción académica? Los datos que se utilizan son de dieciocho universidades e instituciones de educación superior (IES) mexicanas. Las generaciones del estudio comprenden los años que van de 2006 a 2013. Se encontró que los egresados que realizaban producción académica constituían el 13,3\% del total; que el $12,9 \%$ de sus productos contaba con registro de la propiedad intelectual; que el 10,5\% de ellos había participado en proyectos de investigación, había difundido el conocimiento por medio de su participación en conferencias, ponencias y talleres en eventos académicos, había colaborado con colegas y pertenecía al Sistema Nacional de Investigadores en México; que el 51,7\% había impartido clases, y que el 50,9\% había dirigido tesis. Estos resultados determinan la formación en el posgrado por competencias científicas.

Palabras clave: egresados; posgrado; producción académica; perfil profesional; sociedad del conocimiento; ciencia

Resum. La producció acadèmica dels titulats del postgrau d'institucions d'educació superior a Mèxic

La societat del coneixement és una transició de les economies industrials a les economies fonamentades en el saber, basades en inversions en educació, formació, investigació i desenvolupament. D'aquí ve que interessi analitzar la trajectòria de graduació i els perfils professionals dels titulats en base a la producció acadèmica, la satisfacció amb les competències adquirides, amb el programa educatiu i amb la institució, com també, mitjançant l'anàlisi estadística de regressió lineal, donar resposta a la pregunta següent: en quina mesura la trajectòria de graduació es distingeix per la seva productivitat acadèmica? Les dades que s'utilitzen pertanyen a divuit universitats i institucions d'educació superior (IES) mexicanes. L'estudi comprèn els anys que van de 2006 a 2013 i s'hi va trobar que els titulats que realitzaven producció acadèmica constituïen el 13,3\% del total; el 12,9\% dels seus productes estaven registrats a la propietat intellectual; el 10,5\% dels titulats havia 
participat en projectes d'investigació, havia difós el coneixement per mitjà de la seva participació en conferències, ponències, tallers en esdeveniments acadèmics, havia col-laborat amb col-legues i pertanyia al Sistema Nacional d'Investigadors de Mèxic; el 51,7\% havia impartit classes i el 50,9\% havia dirigit tesis. Aquests resultats determinen la formació en el postgrau per competències científiques.

Paraules clau: titulats; postgrau; producció acadèmica; perfil professional; societat del coneixement; ciència

Abstract. The academic production of graduates in postgraduate higher education institutions of Mexico

The knowledge society has marked a transition from industrial economies to knowledgebased economies characterized by investment in education, training, and research and development. In order to determine to what extent the exit pathway of graduates is distinguished by their academic productivity, we analyze the pathways and professional profiles of graduates based on their academic production, as well as satisfaction with the skills acquired, the educational program and the institution. Linear regression analysis was performed to determine the degree to which the career paths of graduates depend on their academic production. Data were collected on eighteen Mexican universities and higher education institutions for the period 2006-2013. It was found that $13.3 \%$ of graduates have academic production; $12.9 \%$ of their production is registered as intellectual property; and $10.5 \%$ have participated in research projects, disseminated knowledge through conferences, presentations, and workshops in academic events, collaborated with colleagues and belongs to the National System of Researchers in Mexico. Moreover, $51.7 \%$ have given classes and $50.9 \%$ have directed theses. These results show that postgraduate training is determined by scientific competencies.

Keywords: graduates; postgraduate studies; academic production; professional profile; knowledge society; science
Sumario
1. Introducción
5. Metodología
2. Objetivo y preguntas de investigación
6. Resultados
3. Fundamentación teórica
7. Comentarios a modo de conclusiones
4. Datos y variables
Referencias bibliográficas

Todo para nosotros y nada para los demás parece haber sido la máxima abominable de los amos de la humanidad de todas las edades del mundo.

(АDAM SMith)

\section{Introducción}

Los episodios extraordinarios en que tienen lugar esos cambios de compromisos profesionales son los que se denominan revoluciones cientificas. Algunos de los cánones del pensamiento científico se enmarcan en los ejemplos más evidentes de revoluciones científicas con los episodios famosos del desarrollo que 
tuvo lugar en dicho ámbito, tales como la física de Aristóteles, el Almagesto de Tolomeo, los principios y la óptica de Newton, la electricidad de Franklin, la química de Lavoisier y la geología de Lyell.

A partir de ello, aparecen las distintas sociedades científicas, tales como la Academia de Lincei en Roma (1600-1630), la Academia del Cimento en Florencia (1651-1657), la Royal Society en Londres (1622) y la Real Academia de Ciencias en París (1960) (Smith, 1972).

En este sentido, cabe preguntarse: ¿qué es la ciencia?, ¿para qué hacer ciencia?, ¿qué es la comunidad científica? De los dos primeros cuestionamientos, se responde que ciencia es la actividad humana creativa, lo cual lleva consigo la aplicación de competencias. Es necesario hacer ciencia para comprender a la naturaleza, y el producto de esa indagación es el conocimiento, al cual se accede mediante un método científico organizado en forma deductiva que aspira a alcanzar consenso entre los expertos desde un compromiso humano (Pérez, 2010). De la última pregunta, se puede decir que una comunidad científica es un grupo social relativamente bien definido. Es una cofradía en la que los individuos se reconocen como miembros del mismo cuerpo (Fourez, 1998).

Por esta razón, como ya apuntó Drucker (1959), la ciencia es la existencia de actividades laborales destinadas al desarrollo del trabajo académico, científico, tecnológico e innovador. Existe una capa social de trabajadores dedicados al conocimiento, por consiguiente, se trata de una sociedad del conocimiento, un espacio para la productividad y el crecimiento intelectual y educativo. De igual forma, Bell (1973), en su trabajo sobre la sociedad posindustrial, habla de un cambio de la economía que genera productos por una economía basada en servicios y cuya estructura está marcada por la preferencia a una clase de profesionales técnicamente cualificada.

El conocimiento ha ocupado siempre el lugar central del crecimiento económico y del aumento progresivo del bienestar social. La capacidad de inventar e innovar, es decir, de crear nuevos saberes y nuevas ideas que se materializan luego en acciones, productos, procedimientos y organizaciones, ha alimentado históricamente al desarrollo humano. A lo largo de todos los tiempos, han existido organizaciones e instituciones eficaces en la creación y en la difusión de conocimientos, desde las corporaciones de la edad media hasta las grandes empresas de comienzos de siglo xx, y desde las abadías cistercienses hasta las academias científicas reales que surgieron a partir del siglo XVII (Foray, 2002).

La sociedad del conocimiento es una transición de las economías industriales a las economías fundadas en el saber, basadas esencialmente en cuantiosas inversiones en materia de educación, formación, investigación y desarrollo, programas informáticos y sistemas de información. Otra de las características de las economías y de las sociedades fundadas en el conocimiento consiste en el uso de las nuevas tecnologías de la información, no solo para la comunicación interpersonal, sino también para la creación de nuevos saberes (Foray, 2002). El conocimiento se ha convertido en la base de los procesos 
productivos, y el tiempo necesario para que un descubrimiento científico se traduzca en aplicaciones tecnológicas es significativamente más corto que en el pasado (Tedesco, 2000).

En este sentido, según Albornoz (2001), América Latina necesita sumarse a los avances de la gran ciencia del conocimiento en ámbitos como las energías alternativas, la aeronáutica, la tecnología espacial, la biotecnología, la microelectrónica, las telecomunicaciones, el tratamiento de la información y los materiales avanzados. Sin embargo, todo ello tiene que sumarse a elementos que deben ser sustanciales, que hacen que las personas sean seres humanos, con valores y sentido de responsabilidad social, por ejemplo: el campo del medio ambiente. Es vital preguntarse cómo se utiliza el conocimiento, a quién sirve y cómo se difunde, e invertir en ciencia básica y aplicada. Es necesario invertir en el trabajo interdisciplinario, además de cuestionar en qué dirección va ese conocimiento, a qué nichos se destina y hacia dónde se dirige el talento visto como capital humano. Asimismo, debemos plantearnos para qué sector es funcional el saber (para la industria, para la ciencia, para la humanidad, para los empresarios...). Ante tales cuestionamientos, es necesario que exista una responsabilidad social y ética de la ciencia.

En ese sentido, la responsabilidad social y ética de la ciencia radicará en prestar atención a la comprensión de las interacciones entre sujetos, y el vínculo con la ciencia debe ampliarse más allá de las comunidades. Entre otras cosas, ello significa relacionar las colectividades científicas agrupadas en instituciones formadas por otros sujetos de la vida colectiva, entre ellas, las clases sociales. Estas, de acuerdo con sus intereses, en primer lugar económicos, y a la luz del proyecto político e ideológico que propugnen, deben definir su posición ante la ciencia, promoviéndola, retardándola, planteándole fines humanitarios o deshumanizados, con lo que conferirán un sentido social o elitista a su acción. La ciencia se presenta, así, como un valor social: ciencia para algo y ciencia para alguien. A ella, se le asigna determinado interés e importancia, se le orienta en una u otra dirección o, simplemente, se le menosprecia. En cualquier caso, se manifiesta una definida proyección valorativa de las clases sociales respecto a la ciencia (Núñez, 2000; Morin, 2004).

Por su parte, salvo algunas excepciones, es típico que las empresas privadas de los países en desarrollo, e incluso las públicas, no tengan áreas de innovación. En particular, las estadísticas referentes a los estados latinoamericanos revelan, acerca del número de los investigadores, la matrícula del posgrado y el gasto público en actividades de investigación y desarrollo, que, en general, tienen una base débil para establecer un sistema de innovación sólido que impacte significativamente en el crecimiento económico. De manera puntual, los derechos de propiedad intelectual y las fuentes de financiamiento para la ciencia y la tecnología son escasos en la mayor parte de la región (Moreno y Ruiz, 2009). Ello es debido a que, en diversos casos, existe poca destinación de recursos, a lo que se suma una carente articulación entre las políticas de impulso a la ciencia en la formación de capital humano y la inserción en ámbitos necesarios para la aplicación de sus conocimientos. 
En este sentido, cabe preguntarse: ¿cómo sería posible reflexionar, concientizar y actuar a través de una relación positiva entre ciencia y sociedad? La respuesta nos vendría dada a través de la formación de competencias en educación y en capital. La ciencia, como todas las demás instituciones sociales, debe ser apoyada por un grupo con compromiso, ethos científico, que es el complejo de normas que todo hombre de ciencia debe considerar como esencial (Popper, 1961; Kuhn, 1962; Merton, 2003; Pérez, 2010).

Ante este contexto histórico e internacional, en el panorama mexicano, el desarrollo de la ciencia en espacios áulicos formales tiene su actuación en la Universidad Nacional Autónoma de México (UNAM), con la Escuela de Altos Estudios, la cual fue la primera institución que ofreció programas de posgrado a partir de 1926 (García, 1995), mientras que la política para el desarrollo y la creación de programas educativos de posgrado se dio con el surgimiento del Consejo Nacional de la Educación Superior y la Investigación Científica (CNESIC), creado en 1935, figura que actualmente es conocida como el Consejo Nacional de Ciencia y Tecnología (CONACYT), creado en 1970.

En el análisis teórico expuesto, radica el fundamento filosófico de la creación de programas educativos y de planes de estudio de posgrado, así como el diseño de los perfiles profesionales, las características y la trayectoria de los egresados, el tipo de trabajo y la producción académica, donde la sociedad del conocimiento señala los cambios en los compromisos profesionales según los requerimientos para el desarrollo de la ciencia y las disciplinas.

\section{Objetivo y preguntas de investigación}

De acuerdo con lo expuesto más arriba, interesa analizar la trayectoria de egreso y los perfiles profesionales de los egresados con base a la producción académica, a la satisfacción con las competencias adquiridas, al programa educativo y a la institución, así como dar respuesta a las preguntas siguientes de investigación: ¿en qué medida la trayectoria de egreso se distingue por su producción académica?; en realidad, ¿’os egresados generan conocimiento de alto nivel?; jexisten diferencias significativas entre el área de conocimiento, el nivel y la orientación del posgrado en la contribución de los egresados al conocimiento, a través de su producción académica, la difusión del saber, las acciones de vinculación entre pares y la formación de recursos humanos?; ¿existen diferencias sustantivas entre la producción académica de acuerdo con la trayectoria de egreso y el perfil profesional?; ¡ cómo se encuentra de satisfecho el egresado con la contribución del posgrado en el desarrollo de competencias adquiridas, con el programa educativo y con la institución del posgrado cursado?, y ¿en qué medida los egresados tienen reconocimiento científico nacional y extranjero? 


\section{Fundamentación teórica}

\subsection{La producción académica de egresados del posgrado}

Para hablar de producción académica, es necesario tener presente el concepto de grupo de pares académicos, dedicados a la generación, a la aplicación y a la difusión de conocimiento en una disciplina o en un área específica del saber. Por tanto, se trata de propiciar nuevas reflexiones, teorías, hipótesis y estudios para mantener una conversación académica y avanzar en una disciplina o en un área específica del conocimiento. Como consecuencia de lo anterior, la producción académica se define como el producto intelectual de las actividades realizadas en la investigación, la docencia y la ciencia (Crane, 1972; Merton, 1985; Burton, 1987; Becher, 2001).

En este sentido, se definió a la producción académica como:

1. Los productos académicos tales como artículos, libros publicados, libros traducidos, libros editados, capítulos de libros, desarrollo tecnológico, innovación, transferencia tecnológica, patentes registradas, citas a las propias publicaciones, memorias de congresos y reseñas.

2. La generación del conocimiento se determinó a partir de los criterios del registro de la propiedad intelectual (por ejemplo: propiedad industrial, copyright, patentes, derechos de autor, ISBN, ISSN, indizado ${ }^{1}$, Information Sciences Institute y arbitrado), así como de la participación en proyectos de investigación.

3. La difusión del conocimiento, en este caso, si el egresado ha participado en eventos académicos (por ejemplo: conferencias, ponencias y talleres).

4. Las acciones de vinculación entre pares o colegas; por ejemplo: si colabora con otros cuerpos académicos de su universidad de adscripción, con otros cuerpos académicos de su país, con otros cuerpos académicos fuera de su país, si participa en cuerpos colegiados de evaluación científica y tecnológica, en cuerpos editoriales o en consultoría, en comisiones dictaminadoras de evaluación de proyectos, en comisiones dictaminadoras en educación superior, en comisiones dictaminadoras de artículos o si pertenece a organismos multilaterales (OCDE, ONU, OEA, BM, OMS). Y también se identifica como las acciones de vinculación entre pares el pertenecer al Sistema Nacional de Investigadores (SNI) en México.

5. La formación de capital humano, si el egresado ha impartido clases y ha dirigido tesis.

1. Indizado en: SCOPUS, SJR, JPR, PubMed, Elsevier, Scirus, Social Sciences Citation Index, Scimago, Agris Internacional, Arts \& Humanities Citation Index, Compludoc, Dialnet, ERIH, Geobase, Geographical Abstracts, Historical Abstracts, IN-RECS, ISOC, Lantindex y RESH, por mencionar algunos. 


\section{Datos y variables}

La representatividad de la muestra se presenta en la tabla 1. En la primera columna, se encuentran las áreas de conocimiento; en la segunda, la muestra de egresados que responden al cuestionario, y en la tercera, el universo de egresados a los que se envió el instrumento.

Las universidades y las Instituciones de Educación Superior (IES) mexicanas participantes en la muestra son dieciocho. Toman parte en ella a partir de planes de estudio incorporados al Programa Nacional de Posgrados de Calidad (PNPC) del CONACYT. Las generaciones del análisis están formadas por egresados entre los años 2006 y 2013, del nivel de especialización, maestría y doctorado, pertenecientes a las áreas de conocimiento siguientes: Físico-Matemáticas, Ciencias de la Tierra, Biología y Química, Humanidades y Ciencias de la Conducta, Ciencias Sociales, Biotecnología y Ciencias Agropecuarias, Medicina y Ciencias de la Salud y Ciencias de la Ingeniería, de las orientaciones en investigación y profesionalizante.

Tabla 1. La representatividad de la muestra de egresados

\begin{tabular}{lrrrcc}
\hline Áreas de conocimiento & \multicolumn{2}{c}{ Muestra } & \multicolumn{2}{c}{ Universo } \\
\hline & $N$ & $\%$ & $N$ & $\%$ \\
\hline Físico-Matemáticas, Ciencias de la Tierra & 16 & 1,40 & 148 & 2,85 \\
\hline Biología y Química & 146 & 12,72 & 522 & 10,01 \\
\hline Humanidades y Ciencias de la Conducta & 802 & 69,92 & 1482 & 28,44 \\
\hline Ciencias Sociales & 66 & 5,75 & 2136 & 40,99 \\
\hline Biotecnología y Ciencias Agropecuarias & 51 & 4,40 & 387 & 7,42 \\
Ciencias de la Ingeniería & 16 & 1,40 & 148 & 2,85 \\
Medicina y Ciencias de la Salud & 50 & 4,40 & 387 & 7,42 \\
\hline Total & 1147 & 100 & 5210 & 100 \\
\hline
\end{tabular}

Fuente: elaboración propia.

Las características representativas de las IES participantes fueron (ver la tabla 2):

1. La clasificación de la calidad de las IES de México es la siguiente: alta = A (10), media = M (2) y baja = B (6).

2. La ubicación geográfica de la institución se encuentra dividida en las partes siguientes: norte $=\mathrm{N}(5)$, centro $=\mathrm{C}(7)$, sur $=S(5)$ y multirregiones $=\mathrm{M}(1)$.

3. Los diferentes tipos de institución son: instituto $=\mathrm{I}(2)$, universidad $=\mathrm{U}$ (14), tecnológico $=\mathrm{T}(1)$ y pedagógica $=\mathrm{P}(1)$.

4. El régimen de la institución se divide en: pública $=\mathrm{PU}(17)$ y privada $=$ PR (1).

Nota: entre paréntesis se detalla el número de casos analizados. 
Tabla 2. Las características de las IES participantes

\begin{tabular}{|c|c|c|c|}
\hline \multicolumn{4}{|c|}{ Códigos } \\
\hline 1. A-C-U-PÚ & 6. A-N-U-PÚ & 11. B-S-U-PÚ & 16. A-C-U-PÚ \\
\hline 2. A-M-I-PÚ & 7. B-S-U-PÚ & 12. A-C-U-PÚ & 17. A-C-P-PÚ \\
\hline 3. M-N-T-PÚ & 8. A-N-U-PÚ & 13. B-N-U-PÚ & 18. M-S-U-PÚ \\
\hline 4. B-N-I-PÚ & 9. A-C-U-PÚ & 14. A-C-U-PR & \\
\hline 5. B-S-U-PÚ & 10. B-S-U-PÚ & 15. A-C-U-PÚ & \\
\hline
\end{tabular}

Fuente: elaboración propia.

La tabla 2 presenta los 18 tipos estudiados, donde por ejemplo A-C-U-PÚ significa, el primer espacio hace referencia a la calidad, así sucesivamente con ubicación, tipo de institución y régimen. A (Calidad: Alta)- C (Ubicación: Centro)- U (Tipo: Universidad)- PÚ (Régimen: Pública).

Esta encuesta se pasó a 5.210 egresados, que son los que pertenecen a dieciocho universidades e instituciones de educación superior (IES) (de los programas participantes: 17 se encuentran en el nivel de doctorado; 25, en el de maestría, y 14, en el de especialización), de estos, contestaron 1147 egresados.

Tal como se observa en la tabla 1, la muestra de egresados está sobrevalorada en el ámbito de Humanidades y Ciencias de la Conducta y menos valorada en el ámbito de Ciencias Sociales.

El cuestionario mide las variables siguientes: características del egresado; programa educativo del posgrado cursado; valoración de la formación recibida; continuación de la formación; trabajo actual que desempeña el egresado y su relación con la formación en el posgrado; posgrado en el ámbito regional, científico, tecnológico y de innovación (la producción académica, divulgación y difusión del conocimiento, las acciones de vinculación entre pares, la formación de capital humano), y nivel propio, adquirido y aplicado de las competencias genéricas del posgrado en el trabajo.

A continuación, las tablas 3 a 6 presentan las variables que se utilizan en el análisis de los datos, con sus correspondientes acrónimos y los valores que representan la presencia o la ausencia de cada característica concreta.

Las variables que se utilizan en cuanto a la trayectoria de egreso son: continuación de la formación, inserción en el mercado laboral, satisfacción sobre el programa educativo y satisfacción respecto a la aplicación de las competencias en el desempeño laboral (véase la tabla 3). 
Tabla 3. Los valores de la variable trayectoria de egreso

\begin{tabular}{|c|c|c|}
\hline $\begin{array}{l}\text { Trayectoria } \\
\text { de egreso }\end{array}$ & Acrónimo & Valor \\
\hline $\begin{array}{l}\text { Continuación } \\
\text { de la formación } \\
\text { (estudios reali- } \\
\text { zados después } \\
\text { del posgrado) }\end{array}$ & CONT_FORM & $\begin{array}{l}1=\text { ha continuado } \\
\text { la formación. } \\
0=\text { no ha continuado } \\
\text { la formación. }\end{array}$ \\
\hline $\begin{array}{l}\text { Inserción en el } \\
\text { mercado laboral }\end{array}$ & INS_MERC_LAB & $\begin{array}{l}1 \text { = está inserto } \\
\text { en el mercado laboral. } \\
0=\text { no está inserto } \\
\text { en el mercado laboral. }\end{array}$ \\
\hline $\begin{array}{l}\text { Satisfacción } \\
\text { sobre el progra- } \\
\text { ma educativo }\end{array}$ & SATISF_DEL_PROG_EDUC & $\begin{array}{l}1=\text { está satisfecho con } \\
\text { el programa educativo. } \\
0=\text { no está satisfecho } \\
\text { con el programa } \\
\text { educativo. }\end{array}$ \\
\hline $\begin{array}{l}\text { Satisfacción } \\
\text { sobre la aplica- } \\
\text { ción de las com- } \\
\text { petencias en } \\
\text { el desempeño } \\
\text { laboral }\end{array}$ & SATISF_APLIC_DE_LAS_COMP_EN_EL_DES_LAB & $\begin{array}{l}1 \text { = está satisfecho } \\
\text { con la aplicación } \\
\text { de las competencias } \\
\text { en el desempeño } \\
\text { laboral. } \\
0=\text { no está satisfecho } \\
\text { con la aplicación } \\
\text { de las competencias } \\
\text { en el desempeño } \\
\text { laboral. }\end{array}$ \\
\hline
\end{tabular}

Fuente: elaboración propia. 
Las variables que se utilizan en cuanto al perfil profesional están relacionadas con las áreas de conocimiento, los niveles y las orientaciones del posgrado (ver la tabla 4).

Tabla 4. Los valores de la variable perfil profesional

\begin{tabular}{|c|c|c|}
\hline Perfil profesional & Acrónimo & Valor \\
\hline Área de conocimiento & A_CON & \\
\hline $\begin{array}{l}\text { Área de conocimiento Físico-Mate- } \\
\text { máticas, Ciencias de la Tierra }\end{array}$ & A_CON_FÍS_MAT_C_TIE & $\begin{array}{l}1 \text { = Físico-Matemáticas, } \\
\text { Ciencias de la Tierra. } \\
0=\text { no Físico-Matemáticas, } \\
\text { Ciencias de la Tierra. }\end{array}$ \\
\hline $\begin{array}{l}\text { Área de conocimiento Biología } \\
\text { y Química }\end{array}$ & A_CON_BIO_QUIM & $\begin{array}{l}1=\text { Biología y Química. } \\
0=\text { no Biología y Química. }\end{array}$ \\
\hline $\begin{array}{l}\text { Área de conocimiento Humanidades } \\
\text { y Ciencias de la Conducta }\end{array}$ & A_CON_HUM_C_COND & $\begin{array}{l}1=\text { Humanidades y Cien- } \\
\text { cias de la Conducta. } \\
0=\text { no Humanidades y } \\
\text { Ciencias de la Conducta. }\end{array}$ \\
\hline Área de conocimiento Ciencias Sociales & A_CON_C_SOC & $\begin{array}{l}1=\text { Ciencias Sociales } \\
0=\text { no Ciencias Sociales } .\end{array}$ \\
\hline $\begin{array}{l}\text { Área de conocimiento Biotecnología } \\
\text { y Ciencias Agropecuarias }\end{array}$ & A_CON_BIOT_C_AGROP & $\begin{array}{l}1=\text { Biotecnología y Ciencias } \\
\text { Agropecuarias. } \\
0=\text { no Biotecnología y Cien- } \\
\text { cias Agropecuarias. }\end{array}$ \\
\hline $\begin{array}{l}\text { Área de conocimiento Ciencias } \\
\text { de la Ingeniería }\end{array}$ & A_CON_C_ING & $\begin{array}{l}1=\text { Ciencias de la Inge- } \\
\text { niería. } \\
0=\text { no Ciencias de la Inge- } \\
\text { niería. }\end{array}$ \\
\hline $\begin{array}{l}\text { Área de conocimiento Medicina } \\
\text { y Ciencias de la Salud }\end{array}$ & A_CON_MED_C_SALUD & $\begin{array}{l}1 \text { = Medicina y Ciencias de } \\
\text { la Salud. } \\
0=\text { no Medicina y Ciencias } \\
\text { de la Salud. }\end{array}$ \\
\hline Nivel del posgrado & N_P & \\
\hline Nivel del posgrado: especialización & N_P_ESPEC & $\begin{array}{l}1=\text { especialización. } \\
0=\text { no especialización. }\end{array}$ \\
\hline Nivel del posgrado: maestría & N_P_MAESTRIA & $\begin{array}{l}1=\text { maestría } \\
0=\text { no maestría }\end{array}$ \\
\hline Nivel del posgrado: doctorado & N_P_DOCTOR & $\begin{array}{l}1=\text { doctorado. } \\
0=\text { no doctorado. }\end{array}$ \\
\hline Orientación del posgrado & O_P & \\
\hline $\begin{array}{l}\text { Orientación del posgrado: } \\
\text { profesionalizante }\end{array}$ & O_P_PROFES & $\begin{array}{l}1=\text { profesionalizante } \\
0=\text { no profesionalizante. }\end{array}$ \\
\hline $\begin{array}{l}\text { Orientación del posgrado: } \\
\text { investigación }\end{array}$ & O_P_INVEST & $\begin{array}{l}1=\text { investigación. } \\
0=\text { no investigación. }\end{array}$ \\
\hline
\end{tabular}

Fuente: elaboración propia. 
Las variables que se utilizan en cuanto a la producción académica son las que se pueden encontrar en la tabla 5 .

Tabla 5. Los valores de la variable producción académica

\begin{tabular}{|c|c|c|}
\hline Producción académica & Acrónimo & Valor \\
\hline La producción académica & PROD_ACAD & $\begin{array}{l}1=\text { sí tiene producción } \\
\text { académica. } \\
0=\text { no tiene producción } \\
\text { académica. }\end{array}$ \\
\hline $\begin{array}{l}\text { La producción académica } \\
\text { tiene propiedad intelectual } \\
\text { o registro }\end{array}$ & PROD_ACAD_PROP_INT_REG & $\begin{array}{l}1=\text { su producción } \\
\text { académica sí tiene } \\
\text { propiedad intelectual } \\
\text { o registro. } \\
0=\text { su producción } \\
\text { académica no tiene pro- } \\
\text { piedad intelectual } \\
\text { o registro. }\end{array}$ \\
\hline $\begin{array}{l}\text { La participación en proyec- } \\
\text { tos de investigación }\end{array}$ & PART_PROY_INV & $\begin{array}{l}1=\text { sí ha participado } \\
\text { en proyectos de inves- } \\
\text { tigación. } \\
0=\text { no ha participado } \\
\text { en proyectos de inves- } \\
\text { tigación. }\end{array}$ \\
\hline $\begin{array}{l}\text { La divulgación y la difusión } \\
\text { del conocimiento }\end{array}$ & DIVULG_Y_DIF_DEL_CON & $\begin{array}{l}1=\text { sí ha divulgado y } \\
\text { ha difundido el conoci- } \\
\text { miento. } \\
0=\text { no ha divulgado ni } \\
\text { ha difundido el conoci- } \\
\text { miento. }\end{array}$ \\
\hline $\begin{array}{l}\text { Las acciones de vincula- } \\
\text { ción entre pares o colegas }\end{array}$ & $\begin{array}{l}\text { ACC_DE_VIN_ENT_PAR_COL_CON_ } \\
\text { COLEGAS }\end{array}$ & $\begin{array}{l}1=\text { sí ha realizado } \\
\text { acciones de vinculación } \\
\text { entre pares o colegas. } \\
0=\text { no ha realizado } \\
\text { acciones de vinculación } \\
\text { entre pares ni colegas. }\end{array}$ \\
\hline $\begin{array}{l}\text { Las acciones de vincula- } \\
\text { ción entre pares desde el } \\
\text { momento de pertenecer } \\
\text { al SNI }\end{array}$ & $\begin{array}{l}\text { ACC_DE_VIN_ENT_PAR_PERT_AL_- } \\
\text { SIST_NAC_DE_INV }\end{array}$ & $\begin{array}{l}1 \text { = sí pertenece al SNI. } \\
0=\text { no pertenece al SNI. }\end{array}$ \\
\hline $\begin{array}{l}\text { La formación de capital } \\
\text { humano: impartir clases }\end{array}$ & FORM_DE_CAP_HUM_IMP_CLAS & $\begin{array}{l}1=\text { sí ha impartido } \\
\text { clases. } \\
0=\text { no ha impartido } \\
\text { clases. }\end{array}$ \\
\hline $\begin{array}{l}\text { La formación de capital } \\
\text { humano: dirigir tesis }\end{array}$ & FORM_DE_CAP_HUM_DIR_TESIS & $\begin{array}{l}1=\text { sí ha dirigido tesis. } \\
0=\text { no ha dirigido tesis. }\end{array}$ \\
\hline
\end{tabular}

Fuente: elaboración propia. 
Las variables que se utilizan en cuanto a la satisfacción son las que se reflejan en la tabla 6.

Tabla 6. Los valores de la variable satisfacción

\begin{tabular}{|c|c|c|}
\hline Satisfacción & Acrónimo & Valor \\
\hline $\begin{array}{l}\text { La satisfacción } \\
\text { de las compe- } \\
\text { tencias en el } \\
\text { desarrollo laboral }\end{array}$ & SATISF_APLIC_DE_LAS_COMP_EN_EL_DES_LAB & $\begin{array}{l}1 \text { = sí está satisfecho } \\
\text { con las competencias } \\
\text { en el desarrollo laboral. } \\
0=\text { no está satisfecho } \\
\text { con las competencias } \\
\text { en el desarrollo laboral. }\end{array}$ \\
\hline $\begin{array}{l}\text { La satisfacción } \\
\text { con el programa } \\
\text { educativo }\end{array}$ & SATISF_INSC_EN_EL_MISMO_PROG_EDUC & $\begin{array}{l}1 \text { = sí está satisfecho } \\
\text { con el programa edu- } \\
\text { cativo. } \\
0=\text { no está satisfecho } \\
\text { con el programa edu- } \\
\text { cativo. }\end{array}$ \\
\hline $\begin{array}{l}\text { La satisfacción } \\
\text { con la institución } \\
\text { del posgrado } \\
\text { cursado }\end{array}$ & SATISF_INSC_EN_LA_MISMA_INST & $\begin{array}{l}1 \text { = sí está satisfecho } \\
\text { con la institución del } \\
\text { posgrado cursado. } \\
0=\text { no está satisfecho } \\
\text { con la institución del } \\
\text { posgrado cursado. }\end{array}$ \\
\hline
\end{tabular}

Fuente: elaboración propia.

\section{Metodología}

La metodología que se utiliza en esta investigación es cuantitativa. En el apartado « En qué medida la trayectoria de egreso se distingue por su producción académica?», por medio del análisis de regresión lineal, se determina el nivel de significación de la variabilidad de la producción académica de los egresados de acuerdo con la trayectoria de egreso.

A través de estadística descriptiva, se determinan las frecuencias y los porcentajes de respuestas de los apartados siguientes: «¿En realidad los egresados generan conocimiento de alto nivel?», «¿En qué medida los egresados tienen reconocimiento científico nacional y extranjero?» y «¿Qué tan satisfecho se encuentra el egresado con la contribución del posgrado en el desarrollo de competencias adquiridas, con el programa educativo y con la institución del posgrado cursado?».

El apartado «¿Existen diferencias significativas entre el área de conocimiento, el nivel y la orientación del posgrado en la contribución de los egresados al conocimiento, a través de su producción académica, difusión del conocimiento, acciones de vinculación entre pares y formación de recursos humanos?» presenta, a partir del análisis de regresión lineal, los niveles de significación con la variable dependiente producción académica y las variables independientes que conforman el concepto del perfil profesional.

Y el apartado "¿Existen diferencias sustantivas entre la producción académica de acuerdo con la trayectoria de egreso y el perfil profesional con base en 
conglomerados?» se determinó por medio de análisis de conglomerados con clústeres jerárquicos.

\section{Resultados}

\section{1. ¿En qué medida la trayectoria de egreso se distingue por su producción académica?}

La producción académica en relación con la trayectoria de egreso se determinó a partir del análisis de regresión lineal, con la variable dependiente producción académica y la variable independiente trayectoria de egreso, que comprende continuación de la formación (estudios realizados después del posgrado), encontrarse inserto en el mercado laboral, satisfacción sobre el programa educativo y satisfacción sobre la aplicación de las competencias en el desempeño laboral.

Así, por ejemplo:

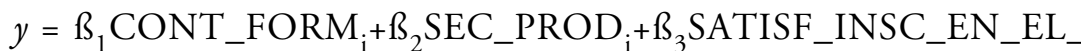

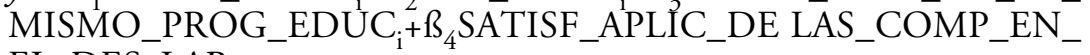
EL_DES_LAB $+v_{\mathrm{i}}$

En donde: $y$ = producción académica de la variable dependiente.

Dicho análisis de regresión lineal permite determinar hasta qué punto la trayectoria de egreso explica la producción académica. Este análisis de regresión lineal se repite para las diferentes variables independientes: continuación de la formación (estudios realizados después del posgrado), encontrarse inserto en el mercado laboral, satisfacción sobre el programa educativo y satisfacción sobre la aplicación de las competencias en el desempeño laboral. En la tabla 7, se resume la significación global de cada regresión lineal.

La trayectoria de egreso se define como los recorridos formativos y laborales que los egresados han seguido a partir de culminar sus estudios en el posgrado. La trayectoria de egreso la medimos con las variables: continuación de la formación (estudios realizados después del posgrado), encontrarse inserto en el mercado laboral, satisfacción sobre el programa educativo y satisfacción sobre la aplicación de las competencias en el desempeño laboral. La variable continuación de la formación determina la trayectoria de egreso relacionada con el hecho de que el egresado tuviera producción académica. De lo cual es posible decir que investigaciones como las de Ruiz (2007) han analizado la continuación de la formación como elemento que coadyuva al ascenso profesional o al acceso a empleos de calidad. En este caso, es posible afirmar que, entre las variables seleccionadas, aquella que determina en gran medida que los egresados tengan producción académica es el hecho de dar continuación a su formación, tal como se aprecia en la tabla 7. 
Tabla 7. La producción académica de los egresados de acuerdo con su trayectoria de egreso

\begin{tabular}{lcc}
\hline Variables & B & Sig. \\
\hline Continuación de la formación & 0,254 & $0,000^{\star * *}$ \\
\hline Sector productivo & $-0,008$ & 0,234 \\
Satisfacción sobre el programa educativo & 0,006 & 0,249 \\
Satisfacción sobre la aplicación de las competencias & $-0,004$ & 0,551 \\
en el desempeño laboral & & \\
\hline
\end{tabular}

Los modelos globalmente significativos se encuentran indicados con un asterisco (al 10\%), con dos asteriscos (al $5 \%$ ) o con tres asteriscos (al 1\%). Aquellos sin asterisco son modelos globalmente no significativos.

Fuente: elaboración propia.

\subsection{En realidad, ¿los egresados generan conocimiento de alto nivel?}

La generación del conocimiento se determinó a partir de los criterios relacionados con la producción académica. Se encontró que los egresados que contaban con producción académica constituían el 13,3\%; de los cuales, un porcentaje de un 12,9\% disponía de propiedad intelectual o de registro (propiedad industrial, copyright, patentes, derechos de autor, ISBN, ISSN, indizado ${ }^{2}$, Information Sciences Institute y arbitrado); un 10,5\% había participado en proyectos de investigación, y con la misma cifra encontramos a quienes habían divulgado y difundido el conocimiento por medio de su participación en conferencias, ponencias, talleres en eventos académicos, habían colaborado con colegas y pertenecían al SNI. Además, el 51,7\% había impartido clases y el $50,9 \%$ había dirigido tesis.

En relación con la producción académica de egresados del posgrado, estos resultados se relacionan con las conclusiones de Jiménez (2014), al señalar que la producción académica es uno de los ejes de análisis que hace evidente la eficacia de un programa doctoral centrado en la formación para la investigación. En su estudio, se consideran unos indicadores similares a estos resultados, tales como la pertenencia a consejos de investigación y al SNI, además de la publicación de artículos en revistas arbitradas y libros (como autores, coordinadores o coautores), así como ponencias y conferencias magistrales en congresos nacionales e internacionales en los últimos tres años.

2. Indizado en: SCOPUS, SJR, JPR, PubMed, Elsevier, Scirus, Social Sciences Citation Index, Scimago, Agris Internacional, Arts \& Humanities Citation Index, Compludoc, Dialnet, ERIH, Geobase, Geographical Abstracts, Historical Abstracts, IN-RECS, ISOC, Lantindex y RESH, por mencionar algunas. 


\section{3. ¿Existen diferencias significativas entre el área de conocimiento, el nivel y la orientación del posgrado en la contribución de los egresados al conocimiento, a través de su producción académica, su difusión del conocimiento, las acciones de vinculación entre pares y la formación de recursos humanos?}

Para dar respuesta a esta pregunta de investigación, se analizó la relación que existía entre el perfil profesional —el cual comprendía el área de conocimiento del posgrado ${ }^{3}$, el nivel de posgrado (especialización, maestría y doctorado) y la orientación (profesionalizante o investigación) - con el hecho de haber creado productos académicos (artículos, libros publicados, libros traducidos, libros editados, capítulos de libros, desarrollo tecnológico, innovación, transferencia tecnológica, patentes registradas, citas a sus publicaciones, memorias de congresos y reseñas) o si había divulgado o difundido el conocimiento (si en el último año participó en eventos académicos, como conferencias, ponencias o talleres).

También era importante analizar si había realizado acciones de vinculación entre pares o colegas; por ejemplo: si colaboraba con otros cuerpos académicos en su universidad de adscripción, con otros cuerpos académicos en su país, con otros cuerpos académicos fuera de su país; si participaba en cuerpos colegiados de evaluación científica y tecnológica, en cuerpos editoriales o de consultoría, en comisiones dictaminadoras de evaluación de proyectos, en comisiones dictaminadoras en educación superior, en comisiones dictaminadoras de artículos; si pertenecía a organismos multilaterales (OCDE, ONU, OEA, $\mathrm{BM}, \mathrm{OMS}$ ), y la relación de la formación de capital humano (si había impartido clases y si había dirigido tesis).

La producción académica en relación con el perfil profesional se determinó a partir del análisis de regresión lineal, con la variable dependiente producción académica y la variable independiente perfil profesional, medida a partir de las variables llamadas área de conocimiento, nivel y orientación del posgrado.

Así, por ejemplo:

$y=\beta_{1} A_{-} C O N_{i}+\beta_{2} N_{-} P_{i}+\beta_{3} O_{-} P_{i}+v_{i}$

En donde: $y$ = producción académica de la variable dependiente.

Dicho análisis de regresión lineal permite determinar hasta qué punto la capacidad explicativa del perfil profesional coadyuva a promover la producción académica. Este análisis de regresión lineal se repite para las diferentes variables independientes: área de conocimiento del posgrado, nivel de posgrado y orientación. En las tablas 8, 9 y 10 se resume su significación global.

El resultado fue que el perfil de egreso (área de conocimiento, nivel de posgrado y orientación) no es determinante para que los egresados realicen pro-

3. Físico-Matemáticas, Ciencias de la Tierra, Biología y Química, Humanidades y Ciencias de la Conducta, Ciencias Sociales, Biotecnología y Ciencias Agropecuarias, Medicina y Ciencias de la Salud y Ciencias de la Ingeniería. 
ducción académica, difusión del conocimiento y acciones de vinculación para formar capital humano. Así lo corroboran los niveles de significación de las tablas 8,9 y 10 .

Tabla 8. La producción académica en relación con el área de conocimiento

\begin{tabular}{lcc}
\hline Variables & B & Sig. \\
\hline Producción académica & $-0,059$ & 0,687 \\
\hline Divulgación o difusión del conocimiento & 0 & 0 \\
Acciones de vinculación entre pares o colaboración entre colegas & $-0,107$ & 0,518 \\
\hline Formación de capital humano al impartir clases & 0,236 & 0,205 \\
\hline Formación de capital humano al dirigir tesis & $-0,122$ & 0,514 \\
\hline
\end{tabular}

Los modelos globalmente significativos se encuentran indicados con un asterisco (al 10\%), con dos asteriscos (al $5 \%$ ) o con tres asteriscos (al 1\%). Aquellos sin asterisco son modelos globalmente no significativos.

Fuente: elaboración propia.

Tabla 9. La producción académica en relación con el nivel de posgrado

\begin{tabular}{lcc}
\hline Variables & B & Sig. \\
\hline Producción académica & 0,031 & 0,735 \\
Divulgación o difusión del conocimiento & 0 & 0 \\
\hline Acciones de vinculación entre pares o colaboración entre colegas & $-0,067$ & 0,516 \\
\hline Formación de capital humano al impartir clases & 0,060 & 0,606 \\
\hline Formación de capital humano al dirigir tesis & $-0,037$ & 0,748 \\
\hline
\end{tabular}

Los modelos globalmente significativos se encuentran indicados con un asterisco (al 10\%), con dos asteriscos (al $5 \%$ ) o con tres asteriscos (al 1\%). Aquellos sin asterisco son modelos globalmente no significativos.

Fuente: elaboración propia.

Tabla 10. La producción académica en relación con la orientación

\begin{tabular}{lcc}
\hline Variables & B & Sig. \\
\hline Producción académica & 0,114 & 0,147 \\
\hline Divulgación o difusión del conocimiento & 0 & 0 \\
Acciones de vinculación entre pares o colaboración entre colegas & $-0,112$ & 0,211 \\
\hline Formación de capital humano al impartir clases & 0,066 & 0,511 \\
\hline Formación de capital humano al dirigir tesis & $-0,015$ & 0,882 \\
\hline
\end{tabular}

Los modelos globalmente significativos se encuentran indicados con un asterisco (al 10\%), con dos asteriscos (al $5 \%$ ) o con tres asteriscos (al 1\%). Aquellos sin asterisco son modelos globalmente no significativos.

Fuente: elaboración propia. 


\section{4. ¿Existen diferencias sustantivas entre la producción académica de acuerdo con la trayectoria de egreso y el perfil profesional con base en conglomerados?}

La diferencia entre la producción académica se determinó con los índices "Producción académica», "Acciones de vinculación entre pares o con colegas», «Conocimiento divulgado o difundido», «Formación de capital humano al impartir clases» y «Formación de capital humano al dirigir tesis», de acuerdo con la trayectoria de egreso (determinada con los índices "Continuación de la formación», «Encontrarse inserto en el ámbito laboral», "Satisfacción para inscribirse en el mismo programa educativo de posgrado" y "Aplicación de las competencias en el ámbito laboral»), así como con el perfil profesional, determinado con los índices "Área de conocimiento", "Nivel y orientación del posgrado cursado».

Todo ello se determinó por medio de análisis de conglomerados, clústeres jerárquicos. De las respuestas, se obtuvo la solución única de tres grupos. Un subgrupo formado por 48 egresados incluyó sujetos que habían realizado acciones de vinculación entre pares o con colegas. Además, habían divulgado y difundido el conocimiento, habían realizado producción académica, habían formado capital humano al impartir clases y habían formado capital humano al dirigir tesis.

Otro subgrupo recoge a 18 egresados, entre quienes el nivel y la orientación del posgrado han sido determinantes para incorporarse al sector productivo y para dar continuación a la formación. Además de un tercer subgrupo con 10 egresados, para ellos, el área de conocimiento cursada del posgrado ha sido importante para la aplicación de las competencias en el desarrollo laboral y para valorar su grado de satisfacción como positivo, al responder que se inscribirían en el mismo programa educativo del posgrado cursado (ver la tabla 11).

Tabla 11. El perfil profesional y la trayectoria de egreso en relación con la producción académica por conglomerados

\begin{tabular}{|c|c|c|}
\hline Subgrupo & Características & $\begin{array}{l}\text { Número } \\
\text { de casos }\end{array}$ \\
\hline 1 & $\begin{array}{l}\text { 1. Acciones de vinculación entre pares o con colegas. } \\
\text { 2. Conocimiento divulgado y difundido. } \\
\text { 3. Producción académica. } \\
\text { 4. Formación de capital humano al impartir clases. } \\
\text { 5. Formación de capital humano al dirigir tesis. }\end{array}$ & 48 \\
\hline 2 & $\begin{array}{l}\text { 1. Nivel del posgrado. } \\
\text { 2. Encontrarse inserto en el ámbito laboral. } \\
\text { 3. Orientación del posgrado. } \\
\text { 4. Continuación de la formación. }\end{array}$ & 18 \\
\hline 3 & $\begin{array}{l}\text { 1. Satisfacción para inscribirse en el mismo programa educativo de } \\
\text { posgrado. } \\
\text { 2. Aplicación de las competencias en el ámbito laboral. } \\
\text { 3. Área de conocimiento. }\end{array}$ & 10 \\
\hline \multicolumn{2}{|c|}{ Total de casos agrupados con base en el total de 1147 egresados encuestados } & 76 \\
\hline
\end{tabular}

Fuente: elaboración propia. 
De lo cual se concluye que la trayectoria de egreso y el perfil profesional en relación con la producción académica es diversa, porque, de un total de 1147 casos, únicamente 76 de ellos son los que corresponden a los conglomerados.

\section{5. ¿En qué medida los egresados tienen reconocimiento científico nacional y extranjero?}

El reconocimiento nacional y extranjero se midió a través de la variable de acciones de vinculación, entendida, por un lado, como la colaboración con colegas en casos como: colabora con otros cuerpos académicos en su universidad de adscripción, colabora con otros cuerpos académicos en su país, colabora con otros cuerpos académicos fuera de su país, participa en cuerpos colegiados de evaluación científica y tecnológica, participa en cuerpos editoriales en consultoría, participa en comisiones dictaminadoras de evaluación de proyectos, participa en comisiones dictaminadoras en educación superior, participa en comisiones dictaminadoras de artículos, pertenece a organismos multilaterales (OCDE, ONU, OEA, BM, OMS), y, por otro, como el hecho de pertenecer al SNI en México. El resultado fue que los egresados que habían realizado acciones de vinculación entendidas como colaboraciones entre colegas y que pertenecían al SNI de México en ambos casos ocupaban el 10,5\%. En este sentido, se encuentran algunas de las investigaciones de Merton (1985) y Bourdieu (1996), las cuales han abundado sobre la meritocracia, el prestigio académico y la reputación basada en los trabajos que el individuo realiza durante su carrera, lo cual ha propiciado un reconocimiento entre pares académicos como lo es en este caso colaborar con colegas y pertenecer al SNI. Estos resultados permiten comprender la inserción laboral en actividades académicas fundamentada en méritos académicos, así como la generación de ofertas académicas de planes de estudios pertinentes y formación en competencias para la continuación de la formación en investigación.

\section{6. ¿Qué tan satisfecho se encuentra el egresado con la contribución del posgrado en el desarrollo de competencias adquiridas, con el programa educativo y con la institución del posgrado cursado?}

La valoración que los egresados hacen de la contribución del posgrado en el desarrollo de competencias adquiridas (en el 27,3\% de los casos, su respuesta es «Muy satisfecho», y en el 49,7\%, su respuesta es «Satisfecho»), con el programa educativo (en el 15,6\% de los casos, su respuesta es "Muy satisfecho", y en el $40,5 \%$, es «Satisfecho») y con la institución del posgrado cursado (en el $27,7 \%$ de los casos, su respuesta es «Muy satisfecho", y en el $42,5 \%$, su respuesta es «Satisfecho») resulta ser positiva. Es necesario determinar los grados de satisfacción de los egresados sobre las competencias, porque, con ese tipo de variables, se está en condiciones de elaborar propuestas como las de investigaciones de Poblete et al. (2016), que presentan estrategias para enseñar y aprender las competencias genéricas. 


\section{Comentarios a modo de conclusiones}

Respecto a la relación de la trayectoria de egreso con la producción académica, algunos de los resultados son los siguientes: se encontró que la regresión lineal no es globalmente significativa entre el hecho de encontrarse inserto en el mercado laboral, la satisfacción sobre el programa educativo y la satisfacción sobre la aplicación de las competencias en el desempeño laboral con el hecho de que el egresado realizara producción académica, pero sí lo es la variable continuación de la formación. Por el contrario, de las variables que se miden del concepto de perfil profesional, ninguna es significativa para que el egresado realice producción académica. En este sentido, Owen (2003) dice que, en la sociedad del conocimiento, la función social de las universidades está cambiando, señala por ejemplo que, en Canadá y en Estados Unidos, las disposiciones legales ejercen presión para exigir a las universidades cada vez mayor relevancia social y económica. Es en este sentido que las instituciones de educación superior pasan de ser transmisoras de conocimiento a ser generadoras de este, así como a ser elementos dinamizadores de la actividad económica a nivel regional, de aquí que sea interesante saber más sobre la producción académica de los egresados de posgrado, de acuerdo con las investigaciones de Gil, Kuri y Franco (1994), Becher (2001), Grediaga (2007) y Figueroa, Bernal y Andrade (2010), que ya han indagado sobre la producción de estudiantes, profesores e investigadores.

En el caso de México, según Santelices (2010), el alto número de investigadores formados a la fecha contrasta con el aún bajo número de patentes obtenidas y la también baja contribución relativa del país al número de publicaciones científicas internacionales y citas de impacto. De aquí que sea necesario continuar con investigaciones que respondan a las cuestiones siguientes: ¿cómo deben involucrarse las políticas para el desarrollo de la ciencia en la sociedad postindustrial?, ¿cómo avanzar de una economía de servicios sin una producción industrial propia?, ¿qué criterios de producción científica deben formularse desde la aún débil presencia del posgrado en América Latina? y ¿cómo fomentar la vinculación entre la docencia y la investigación?, desde ¿para qué? y ¿para quién es esta? En este sentido, es posible afirmar que, de la pregunta de investigación, ¿en realidad, los egresados generan conocimiento de alto nivel?, se encontró que los egresados que realizaban producción académica solamente constituían el 13,3\%, el 12,9\% de sus productos tenían registro de la propiedad intelectual; el $10,5 \%$ de los egresados habían participado en proyectos de investigación y habían difundido el conocimiento por medio de su participación en conferencias, ponencias y talleres en eventos académicos; el 51,7\% había impartido clases y el 50,9\% había dirigido tesis. Estos resultados se relacionan con la investigación de Sandoval (2012), al concluír que, para algunos casos de estudios con egresados, se identifica una carente colaboración en proyectos de investigación, funciones desempeñadas en proyectos de investigación, pertenencia en agrupaciones especializadas y participación en dirección de tesis y tutorías. 
Otro de los resultados fue que el perfil profesional (área de conocimiento, nivel y orientación del posgrado) solo en algunos casos era determinante en la contribución de los egresados al conocimiento, de acuerdo con su producción académica, la difusión del saber, las acciones de vinculación entre pares y la formación de recursos humanos. Hecho que se constata en el análisis de conglomerados con clústeres jerárquicos, al obtener la solución única de tres grupos, lo que determina que sí existen diferencias sustantivas entre la producción académica, de acuerdo con la trayectoria de egreso y el perfil profesional, porque se agrupan únicamente 76 del total de 1147 casos, lo cual permite apreciar la diversidad de tales transiciones. En este sentido, sería interesante proseguir con un análisis posterior, puesto que dicho resultado denota que ciertas competencias científicas del posgrado deben fortalecerse. Investigaciones como las de Jiménez (2014) han concluido que el ámbito del desempeño profesional de los egresados se amplía a partir de la transición de los estudios y en la medida en que cumplen con los rasgos del perfil profesional en los que fueron formados.

Por otra parte, sobre el reconocimiento científico nacional y extranjero de los egresados, determinado por la colaboración con colegas y el hecho de pertenecer al SNI de México, se encontró que únicamente el 10,5\% lo obtiene.

Y, por último, de las preguntas de investigación planteadas sobre ¿qué tan satisfecho se encuentra el egresado con la contribución del posgrado en el desarrollo de competencias adquiridas, con el programa educativo y con la institución del posgrado cursado?, se puede decir que sus respuestas estriban entre "Muy satisfecho» $\mathrm{y}$ "Satisfecho" para los tres casos. Es decir, en cuanto a la valoración de la contribución del posgrado en el desarrollo de competencias adquiridas, los egresados se volverían a inscribir en el mismo programa de posgrado y en la misma institución. Con el tema de la satisfacción de las competencias, se busca destacar una de las conclusiones a las que han llegado la neurociencia y la psicología cognitiva, al recomendar una enseñanza centrada en el estudiante y que este encuentre un sentido y una significación a lo que aprende, al igual que lo han hecho en otros trabajos de investigación, como el de Sánchez, Castañeda, Herrera y Castañeda (2015), al medir la satisfacción con las variables sobre cómo valora el egresado el programa de posgrado, qué tan satisfecho está con la formación adquirida, su satisfacción en relación con el cumplimiento de expectativas y las metas que se propuso al cursar el programa de posgrado.

Es importante estudiar la producción o la productividad académica de egresados, porque determina la trayectoria y la aplicación de perfiles de egreso, así como la utilidad de los perfiles profesionales, lo cual permite estar en condiciones para coadyuvar desde los programas educativos y en el diseño curricular de planes de estudio para la educación de las competencias científicas. Es así que este tipo de investigaciones con base en el análisis de las trayectorias de egreso identifica nuevas formas de concebir el currículo (Tobón, Pimienta y García, 2010) y, de igual manera, nuevos modos de entender los procesos de enseñanza-aprendizaje por competencias. Por ejem- 
plo: en el caso de los docentes, la enseñanza por competencias les plantea enseñar y, al mismo tiempo, aprender también de sus estudiantes, al afirmar que son figuras facilitadoras para la vida, y los estudiantes son vistos como capaces de aprender por sí mismos. En este sentido, es necesario que el alumno busque y genere el conocimiento.

\section{Referencias bibliográficas}

Albornoz, M. (2001). Política científica y tecnológica, una visión desde América Latina. Revista Iberoamericana de Ciencia, Tecnología, Sociedad e Innovación, 1.

BeCHer, T. (2001). Academic tribes and territories. Barcelona: Gedisa.

BELL, D. (1973). The road of the post-industrial society. Madrid: Alianza Universidad. Bourdieu, P. (1996). Las reglas del arte. Madrid: Anagrama.

Burton, C. (1987). The academic profession: National, disciplinary and institutional settings. Berkeley, Los Ángeles: University of California Press.

Crane, D. (1972). Invisible Colleges: Diffusion of Knowledge in Scientific Communities. Illinois: University of Chicago Press.

Drucker, P. (1959). Landmarks of tomorrow. Nueva York: Harper.

Figueroa, S.; Bernal, B. y Andrade, C. (2010). Evaluación de un programa mexicano de Maestría en Psicología desde la perspectiva del egresado: Un estudio sobre los indicadores de calidad. Revista de Educación Superior, 39(153), 23-42.

Foray, D. (2002). La sociedad del conocimiento. Revista Internacional de Ciencias Sociales, 171.

Fourez, G. (1998). La construcción del conocimiento científico: Sociología y ética de la ciencia. Madrid: Narcea.

García, J.M. (1995). El desarrollo del posgrado en México: El caso de los sectores público y privado. Revista Latinoamericana de Estudios Educativos, 20(1), 107-130.

GIL, M.; KURI, R. y Franco, L. (1994). Los rasgos de la diversidad: Un estudio sobre los académicos mexicanos. México: Universidad Autónoma Metropolitana, unidad Azcapotzalco.

GrediagA, R. (2007). Tradiciones disciplinarias, prestigio, redes y recursos como elementos clave del proceso de comunicación del conocimiento: El caso mexicano. Sociológica, 65, 45-80.

JimÉneZ, M. (2014). Trayectorias profesionales de egresados del Doctorado en Educación de la Universidad Autónoma de Tlaxcala: Un análisis de las funciones, productividad y movilidad en el mercado académico. Perfiles Educativos, 36 (143), 30-48.

Kunn, T.S. (1962). The structure of scientific revolutions. Chicago: University of Chicago Press.

Merton, R. (1985). La sociología de la ciencia. Madrid: Alianza.

- (2003). The sociology of science: Theorical and empirical investigations. Chicago: University of Chicago Press.

Moreno-Brid, J.C. y Ruiz, P. (2009). La educación superior y el desarrollo económico en América Latina. México: Comisión Económica para América Latina y el Caribe.

Morin, E. (2004). L'ethique. París: Seuil.

NúNEz, J. (2000). La ciencia y la tecnología como procesos sociales: Lo que la educación científica no debería olvidar. Organización de Estados Iberoamericanos. 
Owen, J. (2003). From separate systems to a hybrid order: Accumulative advantage across public and private science at Research One universities. Research Policy, 32(6), 1081-1104.

Pérez, R. (2010). Reflexiones sobre la ciencia. México: Seminario de Cultura Mexicana.

Poblete, M.; Bezanilla, M.J.; Fernández-Nogueira, D. y Campo, L. (2016). Formación del docente en competencias genéricas: Un instrumento para su planificación y desarrollo. Revista Educar, 52(1), 71-91. <https://doi.org/10.5565/rev/educar.713>

Popper, K.R. (1961). Objective Knowledge. Oxford: Oxford University Press.

Ruiz, E. (2007). Desempeño y reconocimiento laboral del técnico con elevada formación escolarizada. Revista de la Educación Superior, 36(1) 141, 7-21.

Santelices, B. (ed.) (2010). El rol de las universidades en el desarrollo cientifico y tecnológico: Educación Superior en Iberoamérica. Chile: Secretaría General Iberoamericana. Universia. Centro Universitario de Desarrollo (CINDA).

Sánchez, F.; Castañeda, S.; Herrera, J.L. y Castañeda, J. (2015). Estudio de egresados de la Maestría en Ciencias de la Salud. Universidad Autónoma de Zacatecas. Revista de Educación y Desarrollo, 32, 59-65.

SANDoval, R. (2012). Resultados de la encuesta aplicada a egresados del doctorado en historiografía. México: Universidad Autónoma Metropolitana. División de Ciencias Sociales y Humanidades. Unidad Azcapotzalco.

Smith, G.R. (1972). Science and Society: In the sixteen and seventeenth centuries. Londres: Thames and Hudson LTD.

Tedesco, J.C. (2000). Educar en la sociedad del conocimiento. México: Fondo de Cultura Económica.

Tobón, S.; Pimienta, J.H. y García, J.A. (2010). Secuencias didácticas: Aprendizaje y evaluación de competencias. México: Pearson. 